\title{
QR Codes na Educação em Química
}

\author{
Aline Grunewald Nichele - IFRS - Campus Porto Alegre, aline.nichele@ poa.ifrs.edu.br \\ Eliane Schlemmer - UNISINOS, elianes@ unisinos.br \\ Adriana de Farias Ramos - IFRS - Campus Porto Alegre, adrfram@gmail.com
}

\section{RESUMO}

Um número cada vez maior de estudantes possuem dispositivos móveis, como smartphones e tablets, com acesso a internet e possibilidade de instalação de aplicativos com várias funcionalidades. Entre esses aplicativos estão os leitores de códigos de barras bidimensionais, como os $Q R$ Codes, aos quais podem ser associadas informações, sites, vídeos, etc. A aplicação dos $Q R$ Codes no contexto educacional pode instigar a curiosidade dos sujeitos, provocando a ação e a interação, bem como uma nova forma de produzir e acessar a informação. Nesse contexto, apresenta-se uma proposta de uso de $Q R$ Codes no âmbito da Educação em Química. Em especial, foram criados e socializados $Q R$ Codes associados a vídeos contemplando técnicas básicas de laboratório. Eles podem ser disponibilizados "in loco", bem como em papeis, paineis e outros materiais. Com esse trabalho busca-se difundir aos docentes da área o uso educacional dos $Q R$ Codes como uma estratégia de ensino no contexto das tecnologias digitais.

Palavras-chave: $Q R$ Code, telefone celular, tablet

\section{QR Codes in Chemical Education}

\begin{abstract}
An increasing number of students have mobile devices such as smartphones and tablets with internet access and the ability to install applications with different features. Readers of two-dimensional barcodes, such as QR Codes, which can be combined with information, websites, videos, etc., are parts of these applications. QR Codes in educational settings can instigate the curiosity of the subject, stimulating action and interaction as well as a new way to produce and access information. In this context is presented a proposal for the use of QR Codes as part of Chemical Education. Particularly, have been created and socialized QR Codes associated to procedures of basic laboratory technique videos. They can be made available locally as well as in sheets of paper, panels and other materials. In this work it is sought to spread with teachers in this educational area the use of QR Codes as a teaching strategy in the context of digital technologies.
\end{abstract}

Keywords: QR Code, smartphone, tablet 


\section{INTRODUÇÃO}

Smartphones e tablets são dispositivos com crescente adoção entre os estudantes. De acordo com os dados da Pesquisa Nacional por Amostra de Domicílios PNAD de 2011 (IBGE, 2013), o porcentual de pessoas na condição de estudante no Brasil de posse de telefone móvel celular para uso pessoal na população com 10 anos ou mais é de $62,3 \%$, e de utilização da internet é de $65,8 \%$ dos estudantes da rede pública de ensino e de $96,2 \%$ na rede privada. A portabilidade e a mobilidade que smartphones e tablets proporcionam a seus usuários são algumas das características que contribuem para a disseminação e popularidade desses dispositivos.

Segundo Saccol et al. (2011), uma das características proporcionadas por esses dispositivos abrange a mobilidade física, temporal, tecnológica, conceitual e sociointeracional. De acordo com esses autores, a mobilidade física está relacionada aos espaços de aprendizagem oriundos dos momentos de deslocamento físico do estudante. Por sua vez, a mobilidade tecnológica está vinculada aos diferentes dispositivos móveis que $\mathrm{o}$ estudante pode utilizar. A mobilidade conceitual está relacionada às oportunidades e novas necessidades de aprendizagem oriundas da própria mobilidade. A mobilidade sociointeracional está vinculada com a aprendizagem decorrente da interação com diversos níveis e grupos sociais e a mobilidade temporal é aquela caracterizada pela possibilidade de acesso a informação a qualquer momento. No campo da educação, o desenvolvimento de estratégias de ensino e de aprendizagem vinculadas à mobilidade dos aprendizes com o uso de dispositivos móveis com conexão sem fio (levando em consideração os aspectos acima mencionados) impulsionam $\mathrm{o}$ desenvolvimento do mobile learning, ou seja, da aprendizagem com mobilidade.

A crescente adoção de dispositivos móveis como smartphones e tablets pelos estudantes é um fenômeno que desafia as instituições de ensino a incorporá-los no contexto educacional. A partir dessa realidade, uma das possibilidades que emerge é estimular estudantes e professores a trazerem seus próprios dispositivos móveis para as atividades educacionais. A prática do "traga seu próprio dispositivo", conhecida como "Bring Your Own Device" - BYOD (UNESCO, 2014), é recente e se constitui numa via para implementar práticas pedagógicas mediadas por Tecnologias Móveis e Sem Fio (TMSF), baseadas no modelo de aprendizagem 1:1, ou seja, de um dispositivo por estudante, e pode contribuir para ampliar as possibilidades de desenvolvimento do mobile learning.

O contexto do BYOD não está centrado somente nos dispositivos, mas principalmente na possibilidade de conteúdo personalizado que os estudantes acessarão e produzirão por meio deles, de modo que a aprendizagem torna-se mais produtiva. Além disso, a aprendizagem de cada sujeito se dá no ritmo e tempo por ele necessário. $\mathrm{O}$ evento de aprendizagem torna-se único para cada estudante, conferindo-lhe maior autonomia. Por motivos como esses, pesquisadores da área da educação destacam o contexto do BYOD como a melhor prática envolvendo tecnologia para a personalização da aprendizagem (Johnson et al., 2014).

As funcionalidades dos smartphones e tablets podem ser personalizadas e ampliadas por meio da instalação de diversos aplicativos (Apps) disponíveis nas lojas virtuais dos diferentes sistemas operacionais. Entre esses Apps incluem-se os que proporcionam a leitura, bem como a geração de códigos de barras. 


\section{QUICK RESPONSE CODES - QR CODES}

Os Quick Response Codes, conhecidos como QR Codes, são códigos de barras bidimensionais $^{i}$ (Figura 1) que podem ser rapidamente convertidos em informação, por estarem associados a um texto interativo, um link da internet, uma localização geográfica, entre tantas outras possibilidades. O QR Code foi desenvolvido em 1994, pela companhia japonesa Denso Wave Incorporated e sua utilização vem se popularizando mundialmente.

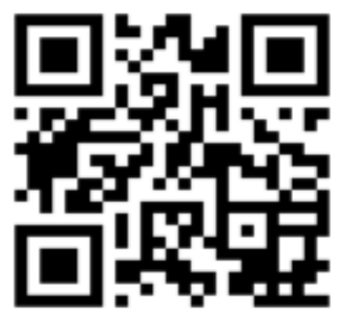

Figura 1 - Exemplo de um $Q R$ Code.

Nos últimos anos eles têm ganhado notoriedade no Brasil pela sua adoção por empresas públicas e privadas de diferentes segmentos. O metrô de São Paulo adotou os $Q R$ Codes para proporcionar aos seus usuários um acesso rápido ao site do metrô, disponibilizando informações culturais, mapas, horários de operação e outros dados sobre o serviço de transporte e regiões atendidas ${ }^{\mathrm{ii}}$. O sistema bancário como, por exemplo, o Banco do Brasil, também tem adotado QR Codes. A maioria dos boletos para pagamento emitidos pelo Banco do Brasil apresenta um $Q R$ Code e com a leitura do mesmo é possível efetuar o seu pagamento ${ }^{\text {iii }}$ diretamente pelo smartphone ou tablet. Jornais impressos, como o gaúcho Jornal Zero Hora, disponibilizam QR Codes com informações adicionais às encontradas em suas folhas impressas.

Recentemente os $Q R$ Codes começaram a ser utilizados também no contexto educacional (Santos et al., 2012; Santos et al., 2010), em diferentes áreas do conhecimento, justamente devido à característica relacionada à facilidade de acesso e difusão da informação.

A leitura e a criação de $Q R$ Codes podem desempenhar importantes papeis no âmbito educacional. Por meio da leitura de $Q R$ Codes o acesso à informação e a interatividade podem ser ampliados. Por outro lado, a criação de $Q R$ Codes pode ser uma interessante estratégia de ensino e de aprendizagem, cujos objetivos podem ser a produção e socialização de materiais, o desenvolvimento da autoria, da autonomia e do trabalho colaborativo.

\section{QR CODES NA EDUCAÇÃO EM QUÍMICA}

Alguns trabalhos relacionados à Educação em Química têm sido desenvolvidos utilizando QR Codes (Battle et al., 2012; Benedict e Pence, 2012; Bonifácio, 2013; Bonifácio, 2012) agregando diferentes possibilidades vinculadas à aprendizagem com mobilidade, mobile learning, no ensino e na aprendizagem de Química.

Battle et al. (2012) criaram uma trilha guiada num jardim da Universidade de Cambridge com 22 pontos de parada, um em cada planta, com a intenção de instigar o interesse dos estudantes pela Química presente na natureza. Em cada ponto de parada os 
visitantes podiam identificar os principais componentes químicos presentes e suas aplicações. Complementarmente, criaram uma trilha digital virtual na qual, por meio de $Q R$ Codes, os visitantes podiam acessar mais informações a respeito dos compostos químicos presentes em cada planta da trilha.

Bonifácio (2012) criou uma tabela periódica dos elementos baseada em $Q R$ Codes. A partir dessa tabela, para cada elemento químico, usando materiais on-line disponíveis gratuitamente, há um áudio relacionado, em língua inglesa, com informações acerca de cada um dos elementos.

O Prêmio Nobel é um dos mais prestigiados prêmios no mundo. Para introduzir o Prêmio Nobel de Química na sala de aula, Bonifácio (2013) construiu um pôster, denominado "QR-NPchem" a partir do qual podem ser acessadas, por meio de $Q R$ Codes, informações sobre cada um dos prêmios concedidos desde a primeira edição em 1901 até a premiação de 2011.

Benedict e Pence (2012) desenvolveram um projeto em que os estudantes são desafiados, na disciplina de Química, a criarem vídeos e fotoblogs relacionados a alguns temas desta ciência. Estes materiais desenvolvidos foram disponibilizados na Web e para cada um deles foi criado um $Q R$ Code. Com esta estratégia, propiciaram aos estudantes o acesso aos materiais criados.

Com a intenção de proporcionar aos estudantes de um curso técnico em Química e de uma Licenciatura em Ciências da Natureza mais subsídios para formá-los no desenvolvimento de atividades e procedimentos inerentes de laboratórios de Química, foram elaborados vídeos com o detalhamento de algumas técnicas básicas de laboratório. Tais vídeos eram socializados com estudantes desses cursos; entretanto, essa socialização era restrita aos estudantes que cursavam as disciplinas que envolviam técnicas básicas de laboratório.

Tendo como objetivo ampliar a disseminação desses materiais a todos os usuários de nossos laboratórios, bem como a todos interessados, incluindo professores e estudantes de diferentes níveis de ensino e instituições, foram criados e associados $Q R$ Codes a esses vídeos, de algumas técnicas básicas de laboratório, os quais são apresentados nesse artigo. Esses códigos de barras bidimensionais podem ser utilizados de diferentes maneiras, entre elas o de serem disponibilizados ao lado de equipamentos como balanças, no caso do detalhamento do procedimento de pesagem, bem como em "roteiros" e orientações de aulas práticas detalhando alguns procedimentos específicos, propiciando aos estudantes maior acesso a informação, maior interatividade e possibilidade de aprender no contexto da mobilidade. Segundo Willians e Pence (2011), a inserção de um desses códigos de barras em uma simples folha de papel torna-a um smart object, ou seja, um "objeto inteligente", pois o conteúdo a ele associado pode ser acessado por meio de um tablet ou smartphone, além também de poder ser alterado dinamicamente.

Com esse artigo busca-se ampliar o acesso e socializar os vídeos criados, a todos os estudantes e docentes de Química e áreas afins, para abordar algumas das "técnicas básicas de laboratório", além de difundir o uso e a criação de $Q R$ Codes na Educação em Química. Entende-se que esses códigos de barras têm potencial para instigar a curiosidade dos sujeitos, provocando a ação e a interação, bem como uma nova forma de produzir e acessar a informação, contribuindo, dessa maneira, para a construção de novas estratégias de ensino e de aprendizagem no contexto das tecnologias digitais. 
Em especial para as técnicas básicas de laboratório de filtração simples, uso de pipetadores, pesagem por adição e preparo de soluções, foram gerados $Q R$ Codes, cada um deles associado a um vídeo com o procedimento da técnica. Nas Figuras 2, 3, 4 e 5 estão, respectivamente, disponibilizados esses $Q R$ Codes.

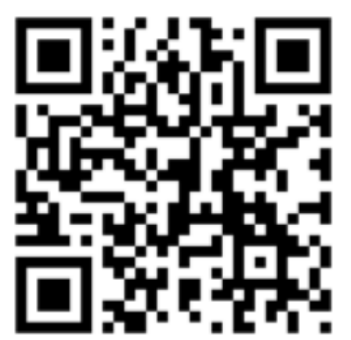

Figura 2 - QR Code associado à filtração simples.

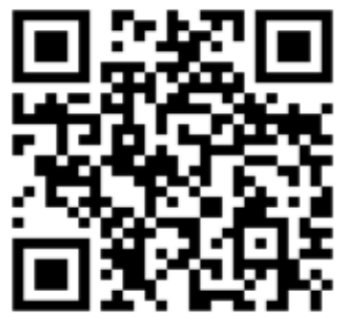

Figura 3 - $Q R$ Code associado ao uso de pipetadores.

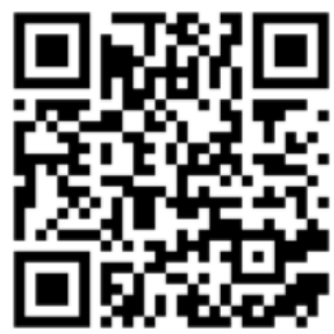

Figura 4 - QR Code associado à pesagem por adição.

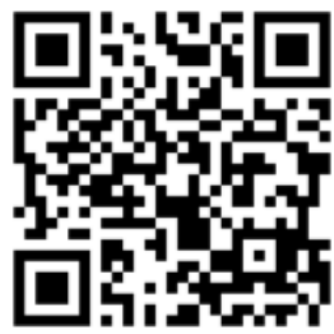

Figura 5 - QR Code associado ao preparo de soluções. 
Os $Q R$ Codes acima podem ser utilizados por todos os estudantes e professores da área de Química ou com interesse nessas técnicas básicas de laboratório. Podem ser disponibilizados nos laboratórios ou em materiais didáticos.

Na educação os $Q R$ Codes podem ser usados em materiais didáticos impressos estando associados a imagens, áudios, vídeos, textos, sites da internet. O conteúdo associado ao $Q R$ Code pode estar disponível na internet, ser criado pelo docente ou, ainda, ser criado pelos estudantes como parte da estratégia de ensino e aprendizagem a ser desenvolvida pelo professor, uma vez que dispositivos como smartphones e tablets capazes de ler $Q R$ Codes provavelmente possuem também as funções de gravação de áudio e vídeo, captura de imagens e acesso a internet, entre outros, o que facilita a autoria de materiais pelos estudantes.

Além dessas possibilidades, os $Q R$ Codes podem estar associados, por exemplo, a blogs que podem ser produzidos e editados pelos estudantes, nesse caso, reconfigurando a prática docente por meio de metodologias e práticas pedagógicas que instiguem a ação e a interação dos sujeitos, bem como o desenvolvimento da autonomia, da autoria, da colaboração e cooperação nos processos de ensino e de aprendizagem.

Os $Q R$ Codes podem ser disponibilizados in loco, ou seja, nos laboratórios junto a equipamentos e bancadas de experimentos (caso dos códigos de barras apresentados nesse artigo), bem como em papeis, paineis e outros materiais.

Com essas possibilidades vinculadas a características como a inexistência de custo para gerar e ler $Q R$ Codes e a facilidade para usá-los, pretendeu-se provocar a curiosidade e o interesse dos professores de Química por essa tecnologia, instigando a descoberta de estratégias inovadoras, no campo das metodologias, das práticas e dos processos de mediação pedagógica.

Nesse sentido, uma das ações promovidas foi a inclusão dos $Q R$ Codes associados aos vídeos de técnicas básicas de laboratório nos roteiros de aulas práticas (Figura 6) de uma disciplina de primeiro semestre de um curso de Licenciatura em Ciências das Natureza: habilitação em Biologia e Química. Dessa maneira, os roteiros de aulas práticas tornaram-se smart objects.

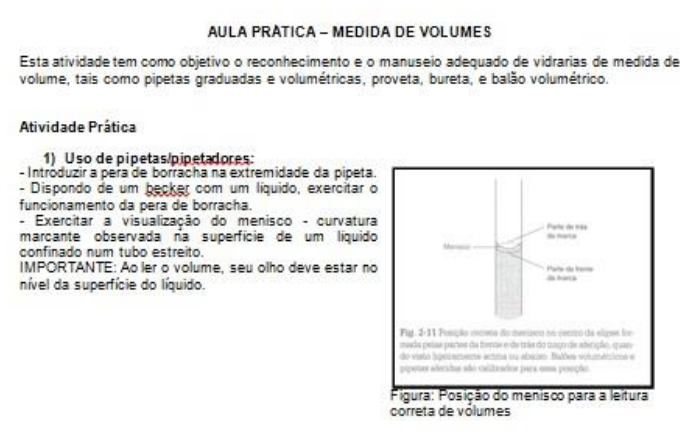

Hara maiores detaines sobre gipetadores eda tecnica de plestagem voce pooe acessar o video "Uso de Ribetadders:"

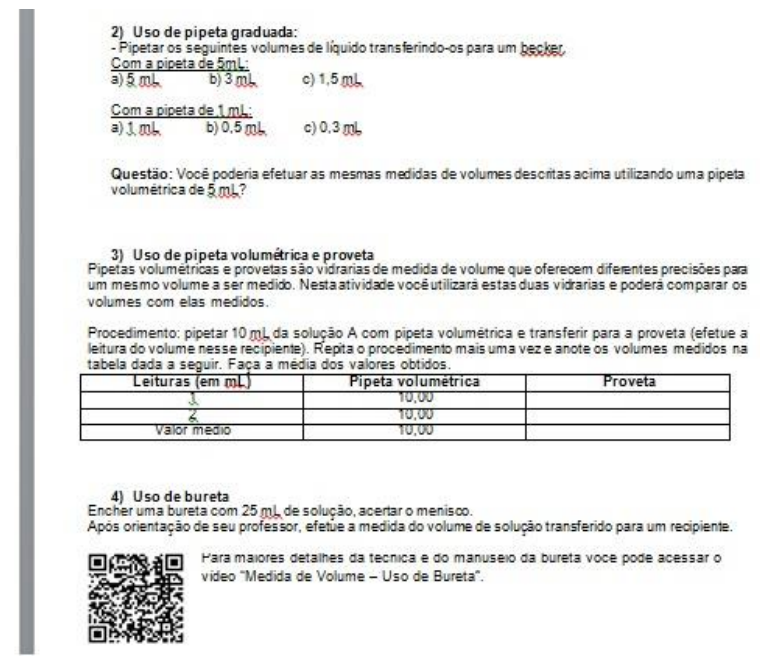

I

Figura 6 - Roteiro de aula prática com $Q R$ Code associado à execução de técnicas básicas de laboratório. 
Todos os 35 estudantes da turma de primeiro semestre dessa Licenciatura em Ciências da Natureza utilizaram o roteiro (Figura 6) para o desenvolvimento da atividade em laboratório. Desses, apenas 6 tinham previamente instalado em seus dispositivos móveis um aplicativo para leitura de $Q R$ Codes. Após a explicação de como deveriam utilizá-los, 8 estudantes instalaram Apps com essa funcionalidade em seus smartphones durante os períodos da aula; outros 16 estudantes instalaram após a atividade prática, com a intenção de poder acessar o conteúdo disponibilizado por meio dos $Q R$ Codes nos roteiros das próximas atividades experimentais.

A introdução dos $Q R$ Codes foi bem aceita pelos estudantes, embora alguns desconhecessem a funcionalidade desses códigos de barras, bem como o procedimento para efetuar sua leitura. Entretanto, todos os estudantes aprovaram a adoção dos $Q R$ Codes e das TMSF no contexto educacional como meio de acesso à informação a qualquer momento, em qualquer lugar.

\section{CONSIDERAÇÕES FINAIS}

Da crescente adoção de dispositivos móveis como smartphones e tablets entre os estudantes e os professores, emerge o potencial desses dispositivos como meio de inovação dos processos de ensino e de aprendizagem em Química, em especial no que se relaciona à ação e à interação dos sujeitos, bem como a forma de produzir, acessar e compartilhar a informação no contexto da mobilidade.

A experiência com esses $Q R$ Codes associados a vídeos de técnicas básicas de laboratório de Química forneceu subsídios, no âmbito das TMSF, para ampliar as ideias e possibilidades de adoção dessas tecnologias nos processos de ensino e de aprendizagem, bem como para modificar a percepção dos estudantes no que tange à inserção das TMSF no contexto educacional, em especial, pela naturalização de adoção dessas tecnologias nos processos de ensino e de aprendizagem, contribuição ampliada em atividades que são desenvolvidas no contexto do BYOD.

Há maior receptividade e aceitação para adoção das TMSF no contexto educacional quando as tecnologias adotadas são familiares aos usuários. Esse é um mérito que pode ser atribuído à perspectiva do BYOD, adotada nesse trabalho, a qual estimula que cada estudante explore e redescubra o potencial de seu próprio dispositivo móvel. Esse é um elemento chave para a adoção das TMSF e da aprendizagem com mobilidade nas instituições de ensino, uma vez que dados censitários apontam para um crescente e significativo avanço do número de estudantes que possuem telefone celular.

Os $Q R$ Codes criados e apresentados nesse artigo podem ser utilizados por toda a comunidade acadêmica pela simples socialização de suas imagens contribuindo para a disseminação do procedimento de técnicas básicas de laboratórios de Química. Além disso, podem servir de inspiração a todos os docentes da área para desenvolverem projetos e atividades educacionais interativas, estimulando a participação, o desenvolvimento da autonomia, da autoria, da colaboração e da cooperação entre os estudantes.

\section{REFERÊNCIAS BIBLIOGRÁFICAS}

BATTLE, G. M.; KYD, G. O.; GROOM, C. R.; ALLEN, F. H.; DAY, J.; UPSON, T. Up the Garden Path: A Chemical Trail through the Cambridge University Botanic Garden. Journal of Chemical Education. n. 89, p. 1390-1394, 2012. 
BENEDICT, L.; PENCE, H. E. Teaching Chemistry Using Student-Created Videos and Photo Blogs Accessed with Smartphones and Two-Dimensional Barcodes. Journal of Chemical Education. n. 89, p. 492-496, 2012.

BONIFÁCIO, V. D. B. Offering QR-Code Access to Information on Nobel Prizes in Chemistry, 1901-2011. Journal of Chemical Education. n. 90, p. 1401-1402, 2013.

BONIFÁCIO, V. D. B. QR-Coded Audio Periodic Table of the Elements: A MobileLearning Tool. Journal of Chemical Education. n. 89, p. 552-554, 2012.

INSTITUTO BRASILEIRO DE GEOGRAFIA E ESTATÍSTICA (IBGE). Pesquisa Nacional por Amostra de Domicílios (PNAD), 2011. IBGE. 2013. Disponível em: < http://www.ibge.gov.br/home/estatistica/populacao/trabalhoerendimento/pnad2011/>.

JOHNSON, L.; ADAMS BECKER, S.; ESTRADA, V.; FREEMAN, A. NMC Horizon Report: 2014 K-12 Edition. Austin, Texas: The New Media Consortium. 2014.

SACCOL, A.; SCHLEMMER, E.; BARBOSA, J. M-learning e u-learning: novas perspectivas das aprendizagens móvel e ubíqua. São Paulo: Pearson Prentice Hall, 2011.

SANTOS, Núbia dos Santos Rosa Santana dos; LIMA, José Valdeni de; WIVES, Leandro Krug. Integração de recursos para acesso aos objetos de aprendizagem multimodais. RENOTE - Novas Tecnologias na Educação, v. 10, n. 3, dezembro, 2012.

SANTOS, Núbia dos Santos Rosa Santana dos; LIMA, José Valdeni de; WIVES, Leandro Krug. Ubiquidade e mobilidade de objetos de aprendizagem usando o papel como recurso. RENOTE - Novas Tecnologias na Educação, v. 8, n. 3, dezembro, 2010 .

UNESCO. The future of mobile learning: implications for policy makers and planners. 2014.2 Disponível em <http://unesdoc.unesco.org/images/0021/002196/219637e.pdf>.

WILlIAMS, A. J.; PENCE, H. E. Smart Phones, a Powerful Tool in the Chemistry Classroom. Journal of Chemical Education. n. 88, p. 683-686, 2011. 
i Todos QR Codes apresentados nesse artigo foram criados utilizando-se o aplicativo "QR Code Creator", disponível na App Store.

${ }^{i i}$ Fonte: http://exame.abril.com.br/tecnologia/noticias/metro-de-sp-adota-qr-code-nas-estacoes. Acesso em: 23 ago. 2014.

${ }^{\text {iii }}$ Fonte: < http://www.bb.com.br/portalbb/page3,101,2183,0,0,1,1.bb>. Acesso em: 23 ago. 2014. 